\title{
1 Hybrid misexpression in multiple developing tissues within a recent adaptive radiation of Cyprinodon pupfishes
}

14 Type: Research Article

$15{ }^{1}$ Department of Biology, University of North Carolina at Chapel Hill, 1250 South Rd., Chapel

16 Hill, NC 27514-3280

17 Running Title: Misexpression in hybrid pupfishes

18 Keywords: RNA-seq, $\mathrm{F}_{1}$ hybrid, misexpression, trophic specialization, allele specific expression,

19 adaptive radiation, transcriptomics

20 Correspondence: Christopher H. Martin. Department of Biology, University of North Carolina at

21 Chapel Hill, Genome Science 2256, 250 Bell Tower Drive., NC, 27514, USA

22 Email: chmartin@unc.edu

23 Word count abstract: 246; Word count main text: 6,594; Tables: 1; Figures: 6 


\section{Abstract}

25 Genetic incompatibilities constitute the final stages of reproductive isolation and speciation, but

26 little is known about incompatibilities that occur within recent adaptive radiations among closely

27 related diverging populations. Crossing divergent species to form hybrids can break up

28 coadapted variation, resulting in genetic incompatibilities within developmental networks

29 shaping adaptive traits. We crossed two closely related sympatric Cyprinodon pupfish species - a

30 dietary generalist and a specialized molluscivore - and measured expression levels in their $\mathrm{F}_{1}$

31 hybrids to identify regulatory variation underlying the novel craniofacial morphology found in

32 this recent microendemic adaptive radiation. We extracted mRNA from eight day old whole-

33 larvae tissue and from craniofacial tissues dissected from 17-20 day old larvae to compare gene

34 expression between a total of seven $\mathrm{F}_{1}$ hybrids and 24 individuals from parental species

35 populations. We found $3.9 \%$ of genes differentially expressed between generalists and

36 molluscivores in whole-larvae tissues and $0.6 \%$ of genes differentially expressed in craniofacial

37 tissue. We found that $2.1 \%$ of genes were misexpressed in whole-larvae hybrids at 8 dpf whereas

$38 \quad 19.1 \%$ of genes were misexpressed in hybrid craniofacial tissue at 17-20 dpf, after correcting for

39 sequencing biases. We also measured allele specific expression across 15,429 phased

40 heterozygous sites to identify regulatory mechanisms underlying differential expression between

41 generalists and molluscivores. Together, our results highlight the importance of considering

42 misexpression as an early indicator of genetic incompatibilities in the context of rapidly diverged

43 morphology and suggests that wide-spread compensatory regulatory divergence drives hybrid

44 misexpression in developing tissues that give rise to novel craniofacial traits. 


\section{Introduction}

53 Changes in gene expression are an important source of variation in adaptive morphological traits

54 (Carroll 2008; Wolf et al. 2010; Indjeian et al. 2016). As genetic variation accumulates in

55 regulatory and coding sequences, stabilizing selection on gene expression results in coevolution

56 such that molecular functions are largely maintained (Coolon et al. 2014; Hodgins-Davis et al.

57 2015). Crossing divergent species to form $F_{1}$ hybrids can break up such coadapted variation,

58 resulting in genetic incompatibilities within developing tissues that give rise to adaptive traits

59 (Michalak and Noor 2004; Landry et al. 2007; Mack and Nachman 2017). Genetic

60 incompatibilities that reduce hybrid fitness can drive reproductive isolation either intrinsically -

61 causing sterility or increased embryonic mortality - or extrinsically whereby incompatibilities

62 reduce hybrid performance in a particular environment (Schluter 2000, Coyne and Orr 2004).

Of particular importance to the process of speciation are genetic incompatibilities caused by hybrid misexpression - when gene expression levels in hybrids are transgressive and fall

65 outside of the range of expression variation observed in both parental species (Michalak and

66 Noor 2004; Ranz et al. 2004; Haerty and Singh 2006; Rockman and Kruglyak 2006; Malone and

67 Michalak 2008; Renaut et al. 2009). This pattern of expression causes Dobzhansky-Muller

68 incompatibilities (DMIs) if incompatible alleles in hybrids cause misexpression that results in

69 reduced hybrid fitness and thus increased post-zygotic reproductive isolation (Presgraves 2003;

70 Coyne 2004; Sweigart et al. 2006; Ortíz-Barrientos et al. 2007; Malone and Michalak 2008;

71 Renaut et al. 2009; Davidson and Balakrishnan 2016). Laboratory studies searching for genes

72 that cause DMIs often identify genes causing sterility or embryonic lethality in hybrids. This

73 approach ignores the fitness consequences of misexpression occurring at later developmental

74 stages within diverse tissue types, thus underestimating the actual number of genetic

75 incompatibilities distinguishing species (Fang et al. 2012; Schumer et al. 2014). Combining

76 findings from these studies with analyses of hybrid misexpression in tissues that give rise to

77 adaptive morphological traits can reveal a broader view of incompatibilities that arise during 78 speciation.

79 Studies of gene expression in hybrids can also implicate regulatory mechanisms

80 underlying expression divergence between parental species, which is important for

81 understanding how expression levels are inherited and how they shape adaptive traits (Wittkopp 
82 et al. 2004; McManus et al. 2010; Mack and Nachman 2017). Research on hybrid gene

83 expression thus far has shown mixed results regarding patterns of inheritance (Signor and

84 Nuzhdin 2018). Some studies found evidence for ubiquitous transgressive expression inherited in

$85 \quad F_{1}$ hybrids (i.e. over- or under-dominance) (Ranz et al. 2004; Rockman and Kruglyak 2006;

86 Roberge et al. 2008), while others found predominately additive patterns (Hughes et al. 2006;

87 Rottscheidt and Harr 2007; Davidson and Balakrishnan 2016). Mechanisms of gene expression

88 divergence in $F_{1}$ hybrids are characterized as interactions between cis-regulatory elements and

89 trans-regulatory factors. Cis elements are often non-coding regions of DNA proximal to genes

90 that are bound by trans-acting proteins and RNAs to regulate mRNA abundance. It is possible to

91 identify mechanisms of gene expression divergence between parental species by bringing cis

92 elements from both parents together in the same trans environment in $\mathrm{F}_{1}$ hybrids and quantifying

93 allele specific expression (ASE) of parental alleles at heterozygous sites (Cowles et al. 2002;

94 Wittkopp et al. 2004). Cis and trans regulatory variants can compensate for one another if

95 stabilizing selection favors an optimal level of gene expression. Hybrid misexpression is

96 expected when different compensatory variants have accumulated in diverging lineages (Denver

97 et al. 2005; Landry et al. 2005; Bedford and Hartl 2009; Goncalves et al. 2012).

98 Here we investigate $\mathrm{F}_{1}$ hybrids from crosses between two closely related species of

99 Cyprinodon pupfishes to understand regulatory mechanisms that led to the evolution of novel

100 craniofacial adaptations in this group (Fig 1A). Cyprinodon brontotheroides - hereafter referred

101 to as the molluscivore - is a trophic specialist species endemic to San Salvador Island, Bahamas

102 that has adapted to eat hard shelled prey including mollusks and ostracods (Martin and

103 Wainwright 2013a,c). This species likely diverged from a generalist common ancestor within the

104 past 10,000 years to occupy this novel niche (Mylroie, J.E, Hagey 1995; Holtmeier 2001; Turner

105 et al. 2008; Martin and Wainwright 2011; Martin 2016). Adapting to this niche involved extreme

106 morphological divergence in craniofacial traits compared to its sympatric generalist sister species

107 Cyprinodon variegatus (Martin and Wainwright 2013c; Lencer et al. 2016). This species

108 consumes mainly algae and detritus and is hereafter referred to as the 'generalist.' Almost all

109 other Caribbean pupfish species are generalists, with the exception of a novel scale-eating

110 pupfish that is also a member of the San Salvador pupfish radiation (Martin and Wainwright

$1112011,2013 c$ ) and a second sympatric radiation of trophic specialists in Laguna Chichancanab,

112 Mexico (Humphries and Miller 1981; Strecker 2006). Molluscivores exhibit a novel skeletal 
113 protrusion on the anteriodorsal head of the maxilla not found in generalist populations that may

114 be used to stabilize prey items held within its oral jaws, which are shorter and more robust

115 relative to generalist species (Fig 1A). This jaw morphology provides higher mechanical

116 advantage for crushing mollusks and other hard-shelled prey (Wainwright and Richard 1995;

117 Martin and Wainwright 2011).

118 Molluscivores and generalists readily hybridize in the laboratory to produce fertile $\mathrm{F}_{1}$

119 offspring with intermediate craniofacial morphologies and no obvious sex ratio distortion

120 (Martin and Wainwright 2011, 2013b; Martin and Feinstein 2014). These species remain largely

121 reproductively isolated in sympatry across multiple lake populations (genome-wide average $\mathrm{F}_{\mathrm{st}}=$

122 0.08; (Martin and Feinstein 2014; West and Kodric-Brown 2015; McGirr and Martin 2017).

123 Therefore, unlike most studies of hybrid misexpression, we are not solely concerned with

124 identifying gene expression patterns underlying hybrid sterility or lethality. Rather, we also aim

125 to characterize misexpression in a developing tissue that gives rise to novel craniofacial

126 phenotypes within a young species pair with ongoing gene flow. We dissected craniofacial tissue

127 from 17-20 day old $\mathrm{F}_{1}$ hybrids and extracted total mRNA to quantify gene expression levels. We

128 also extracted whole-larvae mRNA from 8 day old generalists, molluscivores, and their hybrids.

129 We found misexpression in hybrids at both stages. Finally, we quantified allele specific

130 expression (ASE) across exome-wide phased heterozygous sites to uncover mechanisms of

131 regulatory divergence and found evidence for compensatory variation influencing patterns of

132 hybrid misexpression.

134 Materials and Methods

\section{Study system and sample collection}

136 Our methods for raising larvae and extracting RNA were identical to previously outlined

137 methods (McGirr and Martin 2018). We collected fishes for breeding from three hypersaline

138 lakes on San Salvador Island, Bahamas (Little Lake, Osprey Lake, and Crescent Pond) using a

139 hand net or seine net between 2011 and 2017 . These fishes were reared at $25-27^{\circ} \mathrm{C}, 10-15 \mathrm{ppt}$

140 salinity, $\mathrm{pH} 8.3$, and fed a mix of commercial pellet foods and frozen foods. All lab bred larvae

141 were raised exclusively on newly hatched brine shrimp after hatching and before euthanasia. 
142 Individuals were euthanized in an overdose of buffered MS $\square 222$ and stored in RNA later

143 (Ambion, Inc.) at $-20^{\circ} \mathrm{C}$ for up to 18 months. We used RNeasy Mini Kits (Qiagen catalog

144 \#74104) to extract RNA from all samples.

145 We previously generated 24 transcriptomes belonging to generalists and molluscivores 146 collected at two early developmental stages: 8-10 days post fertilization and 17-20 dpf (McGirr 147 and Martin 2018). RNA was extracted from whole-larvae tissue at 8-10 dpf. We dissected all 1714820 dpf samples to extract RNA from anterior craniofacial tissues containing the dentary, angular, 149 articular, maxilla, premaxilla, palatine, and associated craniofacial connective tissues (Fig. S1).

150 Dissections were performed using fine $\square$ tipped tweezers washed with RNase AWAY (Molecular 151 BioProducts). These 24 samples were generated by breeding populations of lab-raised fishes that 152 resulted from either one or two generations of full-sib breeding between wild caught fishes from 153 Little Lake and Crescent Pond on San Salvador Island, Bahamas (Table 1). There was variation 154 in sampling time because eggs were fertilized naturally within breeding tanks and collected on 155 the same day or subsequent day following egg laying. We collected larvae in a haphazard 156 manner over multiple spawning intervals and it is unlikely that sampling time varied consistently 157 by species.

Here we analyze an additional 19 transcriptomes from generalists, molluscivores, and 159 their hybrids (Table 1). First, we crossed a generalist female with a molluscivore male to 160 generate four $\mathrm{F}_{1}$ hybrids that were collected at 17-20 dpf and extracted RNA from dissected 161 craniofacial tissues. A lab-reared female generalist was used to generate hybrids that was derived 162 from wild caught generalists from Little Lake following one generation of full-sib mating. A lab163 reared male molluscivore was used to generate hybrids that was derived from wild caught 164 molluscivores from Little Lake following two generations of full-sib mating.

165 We performed separate crosses to collect larvae at exactly 8 dpf (190-194 hours after 166 fertilization rather than 8-10 days). We crossed a generalist female with a molluscivore male to 167 generate three $\mathrm{F}_{1}$ hybrids for whole-larvae RNA extractions. The parents of these hybrids were 168 wild-caught from Osprey Lake. Finally, we extracted whole-larvae RNA from six generalists and 169 six molluscivores collected at $8 \mathrm{dpf}$. These samples were generated from wild-caught individuals 170 from Osprey Lake and Crescent Pond. In total, we analyzed transcriptomes from 43 individuals 171 that involved four separate rounds of sequencing (Table 1 and S1). 


\section{RNA sequencing and alignment}

174 The previously reported 24 transcriptomes were sequenced at the High Throughput Genomic

175 Sequencing Facility at UNC Chapel Hill in April 2017 (McGirr and Martin 2018). The 24

176 libraries were prepared at the facility using the KAPA stranded mRNA $\square$ seq kit (KAPA

177 Biosystems 2016) followed by sequencing on one lane of Illumina 150 paired-end Hiseq4000

178 (Table 1 and 2).

19 additional transcriptomes were sequenced at The Vincent J. Coates Genomics

180 Sequencing Laboratory at the University of California, Berkeley. All 19 libraries were prepared

181 at the facility using the Illumina stranded Truseq RNA kit (Illumina RS-122-2001) and all

182 sequencing was performed on Illumina 150 paired-end Hiseq4000. Four libraries for RNA

183 extracted from 17-20 dpf hybrid craniofacial tissues were pooled on a single lane and sequenced

184 in June 2017. 15 libraries for whole-larvae RNA samples collected at exactly 8 dpf were pooled

185 across one and three lanes and sequenced in May $(n=9)$ and July $(n=6) 2018$, respectively

186 (Table 1 and S1).

We filtered all raw reads using Trim Galore (v. 4.4, Babraham Bioinformatics) to remove

188 Illumina adaptors and low $\square$ quality reads (mean Phred score < 20) and mapped filtered reads to

189 the scaffolds of the Cyprinodon reference genome (NCBI, C. variegatus annotation release 100,

190 total sequence length $=1,035,184,475$; number of scaffolds $=9259$, scaffold $\mathrm{N} 50=835,301$;

191 contig N50 = 20,803; (Lencer et al. 2017)) using the RNA $\square$ seq aligner STAR with default

192 parameters (v. 2.5 (Dobin et al. 2013)). We used the featureCounts function of the Rsubread

193 package (Liao et al. 2014) requiring paired $\square$ end and reverse stranded options to generate read

194 counts across 24,952 previously annotated features (Lencer et al. 2017) with an average coverage

195 depth of 136 reads (Table S2 and S3).We assessed mapping and count quality using MultiQC

196 (Ewels et al. 2016). We previously showed that there was no difference between generalists and

197 molluscivores in the proportion of reads that map to annotated features of the Cyprinodon

198 reference genome (McGirr and Martin 2018). Similarly, here we found no difference in the

199 proportion of reads mapping to features between generalists, molluscivores, and hybrids (Fig.

200 S2; ANOVA, $P=0.6$ ), but we did find that fewer reads mapped to features in 17-20 dpf samples 201 than $8 \mathrm{dpf}$ samples (ANOVA, $P=2.38 \times 10^{-10}$ ). 
Since we analyzed RNA from 43 individuals that were sequenced across four different dates and their libraries were prepared with either KAPA or TruSeq stranded mRNA-seq kits, we tested whether a significant amount of between-sample variance in read counts was explained by sequencing date or library preparation kit. We fit linear models (using the $\operatorname{lm}($ ) function in $\mathrm{R}$ ) to determine whether normalized counts across individuals were influenced by 1) the number of PCR duplicate reads produced during sequence amplification, 2) the uniformity of coverage across a transcript, or 3) the depth of coverage across GC-rich transcripts. All of these measures could have been influenced by different library preparation methods (Alberti et al. 2014; Van

210 Dijk et al. 2014; KAPA Biosystems 2016). We quantified the number of duplicate reads and the

211 median percent GC content of mapped reads for each sample using RSeQC (Wang et al. 2012).

212 We also used RSeQC to estimate transcript integrity numbers (TINs) which is a measure of 213 potential in vitro RNA degradation within a sample. TIN is calculated by analyzing the

214 uniformity of coverage across transcripts. (Wang et al. 2012, 2016). We performed ANOVA to 215 determine whether the proportion of duplicate reads, GC content of reads, TINs, the number of 216 normalized read counts, number of raw read counts, or number of raw fastq reads differed

217 between samples grouped by library preparation method and by sequencing date.

\section{Differential expression analyses and hybrid inheritance of expression patterns}

220 We performed differential expression analyses with DESeq2 (v. 3.5 (Love et al. 2014)). This

221 program fits negative binomial generalized linear models for each gene across samples to test the

222 null hypothesis that the fold change in gene expression between two groups is zero. DESeq2 uses 223 an empirical Bayes shrinkage method to determine gene dispersion parameters, which models

224 within-group variability in gene expression, and logarithmic fold changes in gene expression.

225 DESeq2 normalizes raw read counts by calculating a geometric mean of counts for each gene 226 across samples, dividing individual gene counts by this mean, and using the median of these

227 ratios as a size factor for each sample. These sample-specific size factors account for differences

228 in library size and sequencing depth between samples. Gene features showing less than 10

229 normalized counts in every sample in each comparison were discarded from analyses.

230 Differential expression between groups was determined with Wald tests by comparing

231 normalized posterior log fold change estimates and correcting for multiple testing using the 
232 Benjamini-Hochberg procedure with a false discovery rate of 0.05 (Benjamini and Hochberg

233 1995). We also used DESeq2 to perform clustering and principal component analyses (Fig. S3).

234 We conducted pairwise comparisons to identify genes differentially expressed between

235 hybrids vs. parental species, hybrids vs. generalists, hybrids vs. molluscivores, and generalists

236 vs. molluscivores. "Parental species" refers to generalists and molluscivores derived from the

237 same populations as the parents of the hybrid samples. We did not sequence any of the parents

238 crossed to generate hybrids. We defined genes as misexpressed in hybrids if they were

239 significantly differentially expressed between hybrids and the parental species samples. First, we

240 compared whole-larvae gene expression between samples collected at $8 \mathrm{dpf}$ (six generalists, six

241 molluscivores, and three hybrids). All of the $8 \mathrm{dpf}$ samples were sequenced at the Vincent $\mathrm{J}$.

242 Coates Genomic Sequencing Laboratory, University of California Berkeley (VJCGSL UCB) and

243 their libraries were all prepared using the TruSeq stranded mRNA-seq kit. Second, we compared

244 craniofacial tissue gene expression between samples collected at 17-20 dpf (six generalists, six

245 molluscivores, and four hybrids). The generalist and molluscivore samples were sequenced at the

246 High-Throughout Sequencing Facility, University of North Carolina Chapel Hill (HTSF UNC)

247 and their libraries were prepared using the KAPA stranded mRNA-seq kit, while the hybrids

248 were sequenced at the VJCGSL UCB and their libraries were prepared using the TruSeq kit. In

249 order to understand how sequencing at different facilities and using different library prep

250 methods affected the proportion of genes misexpressed between hybrids and parental species at

251 17-20 dpf, we performed a third set of comparisons between hybrids collected at $8 \mathrm{dpf}$

252 (sequenced at VJCGSL UCB with TruSeq) and generalists and molluscivores from a previous

253 study collected at 8-10 dpf (sequenced at HTSF UNC with KAPA; (McGirr and Martin 2018)).

254 We measured how many genes were differentially expressed between 8 dpf hybrids vs. 8-10 dpf

255 parental species than there were differentially expressed between $8 \mathrm{dpf}$ hybrids vs. $8 \mathrm{dpf}$ parental

256 species. This allowed us to estimate an upper-limit on the proportion of genes falsely identified

257 as differentially expressed between 17-20 dpf hybrids and 17-20 dpf parental species due to

258 samples being sequenced at different facilities with different library preparation kits.

259 To determine whether genes showed additive, dominant, or transgressive patterns of

260 inheritance, we quantified differences in gene expression between hybrids vs. parental species

261 and compared them to genes differentially expressed between generalists vs. molluscivores (Fig. 
2). Hybrid inheritance was considered additive if hybrid gene expression was intermediate between generalists and molluscivores with significant differential expression between generalists and molluscivores, respectively. Inheritance was dominant if hybrid expression was significantly different from one parent species but not the other. Genes showing misexpression in hybrids showed transgressive inheritance, meaning hybrid gene expression was significantly higher (overdominant) or lower (underdominant) than both parental species.

\section{Gene ontology enrichment analyses}

270 The Cyprinodon reference genome is annotated for genomic features (NCBI, C. variegatus

271 Annotation Release 100, (Lencer et al. 2017)), and many annotated genes share the same name

272 as their zebrafish orthologs. We performed gene ontology (GO) enrichment analyses for genes

273 differentially expressed between species and misexpressed in hybrids that shared the same name

274 as zebrafish orthologs using GO Consortium resources available at geneontology.org (Ashburner

275 et al. 2000; GO Consortium 2017). We searched for enrichment across biological process

276 ontologies curated for zebrafish.

\section{Allele specific expression and mechanisms of regulatory divergence}

279 We followed the best practices guide recommended by the Genome Analysis Toolkit (DePristo

280 et al. 2011 (v. 3.5)) in order to call and refine SNP variants within coding gene regions using the

281 Haplotype Caller function. We called SNPs across all filtered reads mapped to annotated features

282 for 17-20 dpf samples and $8 \mathrm{dpf}$ samples using conservative hard-filtering parameters (DePristo

283 et al. 2011; Marsden et al. 2014): Phred-scaled variant confidence divided by the depth of

284 nonreference samples $\square>2.0$, Phred-scaled $P$-value using Fisher's exact test to detect strand

285 bias $\square>\square 60$, Mann-Whitney rank-sum test for mapping qualities (z $\square>\square$ 12.5), Mann-Whitney

286 rank-sum test for distance from the end of a read for those with the alternate allele

$287 \quad$ ( $\square>\square 8.0$ ). We used the ReadBackedPhasing function with a phase quality threshold $>20$ to

288 identify phased variants. We used the VariantsToTable function (with genotypeFilterExpression

289 "isHet ==1") to output phased heterozygous variants for each individual. We counted the

290 number of reads covering heterozygous sites using the ASEReadCounter (with -U 
ALLOW_N_CIGAR_READS -minDepth 20 --minMappingQuality 10 --minBaseQuality 20 -drf DuplicateRead). In total we identified 15,429 phased heterozygous sites across all 32 individuals with sequencing coverage $\geq 20 \times$ that fell within 3,974 genes used for differential expression 294 analyses.

We assigned each heterozygous allele as the reference allele, alternate allele, or second 296 alternate allele and matched each allele to its corresponding read depth. This allowed us to 297 identify allele specific expression (ASE) by measuring expression variation between the two 298 haplotypes of each gene distinguished by heterozygous sites. We used a binomal test in $\mathrm{R}$ 299 (binom.test) to determine if a heterozygous site showed significantly biased expression of one 300 allele over another $(P<0.05$; McManus et al. 2010; Mack and Nachman 2016). We measured ASE across 3,974 genes expressed in parental species and hybrids. A gene was considered to

302 show ASE in hybrids if a phased heterozygous allele within that gene showed consistent biased 303 expression in all hybrid samples (17-20 dpf $n=4 ; 8 \mathrm{dpf} n=3)$ and did not show ASE in any of 304 the parental samples ( $\mathrm{n}=12$ for both developmental stages).

Gene expression controlled by compensatory variation in parental species is often associated with misexpression in their hybrids (Landry et al. 2005, 2007; Bedford and Hartl 2009; Goncalves et al. 2012). Regulatory elements that have opposite effects on the expression level of a particular gene can compensate for one another to produce an optimal level of gene expression favored by stabilizing selection (Denver et al. 2005; Goncalves et al. 2012). Diverging species can evolve alternate compensatory mechanisms while maintaining similar expression levels (True and Haag 2001). Hybrids of such species would have a mismatched

313 result in biased expression of parental alleles (Wittkopp et al. 2004; Landry et al. 2005). Thus,

314 we identified gene expression controlled by compensatory regulatory variation if a gene 1) did 315 not show differential expression between generalists and molluscivores, 2) showed significant

316 ASE at one or more heterozygous sites in hybrids, and 3) did not show ASE at any site in

317 generalists or molluscivores. Finally, we looked for overlap between genes showing

318 compensatory regulation and genes showing misexpression in hybrids.

\section{Results}




\section{Differential expression between generalists and molluscivores}

322 We previously found 1,014 genes differentially expressed in whole-larvae tissue between six 323 generalists and six molluscivores collected at 8-10 dpf (McGirr and Martin 2018). Here we

324 compared gene expression in whole-larvae tissue collected at exactly 8 dpf (190-194 hours after

325 fertilization rather than 8-10 dpf) between six generalists and six molluscivores. We found 700

326 out of 17,723 (3.9\%) genes differentially expressed between species (Fig 1C). 235 of the 700

327 genes were annotated as zebrafish orthologs and used for gene ontology enrichment analyses.

328 Encouragingly, the only significantly overrepresented ontology was skeletal system

329 morphogenesis (GO:0048705) which matched 11 differentially expressed genes (Table S4).

330 We previously found 120 genes differentially expressed in craniofacial tissue between

331 species at 17-20 dpf (McGirr and Martin 2018). Here we reexamined gene expression in those

332 same individuals using a more conservative threshold for genes to be included in differential

333 expression analyses (where a gene must show $>=10$ normalized counts in every sample included

334 in the comparison). As expected, we found fewer genes differentially expressed using this more

335 conservative threshold (81 out of 13,901 (0.6\%); Fig 1E). These 81 genes did not show

336 enrichment for any biological process ontologies.

\section{Hybrid misexpression in whole-larvae tissue}

339 We compared gene expression in whole-larvae tissue collected at $8 \mathrm{dpf}$ from generalist and

340 molluscivore populations $(n=12)$ with expression in their $F_{1}$ hybrids $(n=3)$ and found that 370

341 out of 17,705 genes (2.1\%) were misexpressed in hybrids (Fig. 1D). Slightly more genes showed

342 underdominant inheritance $(209 ; 1.2 \%)$ than overdominant inheritance $(154 ; 0.89 \%$; Fig. 3A and

343 C). The magnitude of differential expression was higher for genes showing underdominant

344 inheritance than overdominant inheritance (Fig. S4; Wilcoxon rank sum test, $P=8.5 \times 10^{-5}$ ). Of

345 the 370 genes showing misexpression, 138 were annotated as zebrafish orthologs used for gene

346 ontology enrichment analyses. The only significantly overrepresented term was cellular lipid

347 metabolic process (GO:0044255).

348 The majority of genes showed conserved levels of expression with no significant 349 difference between hybrids and parental species (84.9\%). In line with other hybrid expression 
studies (Hughes et al. 2006; Rottscheidt and Harr 2007; Davidson and Balakrishnan 2016), most genes that did not show conserved inheritance showed additive inheritance $(399 ; 2.3 \%)$. We

352 found some genes showing evidence for dominance, with $89(0.51 \%)$ showing 'generalist-like'

353 expression patterns and $168(0.97 \%)$ showing 'molluscivore-like' patterns of inheritance (Fig 3A 354 and C).

\section{Hybrid misexpression in craniofacial tissue}

357 We compared gene expression in craniofacial tissue collected at 17-20 dpf from generalist and 358 molluscivore populations $(n=12)$ with expression in their $F_{1}$ hybrids $(n=4)$ and found extensive 359 hybrid misexpression. More than half of genes (6,590 out of $12,769(51.6 \%))$ were differentially 360 expressed in hybrids compared to parental species expression (Fig 1F). There was an 361 approximately equal number of genes showing overdominant and underdominant expression in 362 hybrids, with 3,299 (25.83\%) genes showing higher expression in hybrids relative to parental 363 species and 3,291 (25.77\%) showing lower expression in hybrids (Fig 1F, Fig 3B and D). While 364 there was a similar number of genes showing over- and underdominance, the magnitude of 365 differential expression was higher for genes showing underdominance (Fig. S4; Wilcoxon rank 366 sum test, $P<2.2 \times 10^{-16}$ ). Of the 6,590 genes showing misexpression, 2,876 were annotated as

367 zebrafish orthologs used for gene ontology enrichment analyses. Misexpressed genes were 368 enriched for 210 ontologies, including embryonic cranial skeleton morphogenesis (GO:0048701;

369 Table S5 and S6).

Hybrid misexpression is influenced by library preparation and sequencing conditions

372 All of the $8 \mathrm{dpf}$ samples were sequenced at the same facility using the same library preparation

373 kit. However, the 17-20 dpf generalist and molluscivore samples were sequenced at a different

374 facility than the 17-20 dpf hybrid samples and used a different library preparation kit. We took

375 two approaches toward understanding how sequencing at different facilities and using different

376 library kits may have affected the proportion of genes misexpressed between hybrids and

377 parental species at 17-20 dpf. 
First, we performed another differential expression comparison between whole-larvae hybrids collected at $8 \mathrm{dpf}$ and whole-larvae parental species that we collected for a previous study between 8-10 dpf (McGirr and Martin 2018). The $8 \mathrm{dpf}$ hybrids were sequenced at the same facility with the same library kit as the 17-20 dpf hybrids, while the 8-10 dpf parental species were sequenced at the same facility with the same library kit as the 17-20 dpf parental species. This design mirrored the comparison we used to estimate 17-20 dpf hybrid craniofacial misexpression, but at an earlier developmental stage (Fig. S5). Whereas comparisons between 8 dpf hybrids and parental species sequenced under the same conditions revealed 370 genes $(2.1 \%)$ misexpressed, comparisons between hybrids and parental species sequenced at different sequencing centers with different library preparation kits suggested that 997 (6\%) genes were misexpressed - a 37\% increase (Fig. S5). This presents a major caveat to our findings, but does not suggest that all genes showing hybrid misexpression in 17-20 dpf craniofacial tissues are false-positives. Using this estimate of bias to correct for different library preparation methods for our 17-20 dpf samples, we estimate that $19.1 \%$ genes were misexpressed in hybrid craniofacial tissue rather than $51.6 \%$.

We also investigated whether a significant amount of between-sample variance in read

394 counts was explained by library preparation method or sequencing date. For each sample we 395 quantified the number of normalized read counts, raw read counts, and raw fastq reads. We also

396 estimated the proportion of duplicate reads out of total mapped reads, the median percent GC

397 content across mapped reads, and the uniformity of coverage across mapped reads (median 398 transcript integrity numbers (TINs)). All of these measures could be influenced by different 399 library preparation methods (Alberti et al. 2014; Van Dijk et al. 2014; KAPA Biosystems 2016).

400 Library preparation method was not associated with differences in the number of normalized 401 read counts or median TINs (Fig. $4 \mathrm{~A}$ and B; Welch two sample t-test, $P>0.05$ ). When we 402 grouped samples by sequencing date rather than library preparation method, we found that the 403 17-20 dpf hybrid craniofacial samples (sequenced 6/17) did not show any difference in median 404 GC content, raw read counts, or raw fastq reads compared to samples sequenced on different 405 dates (Fig S6). However, these samples did show lower proportions of duplicate reads, fewer 406 normalized read counts, and lower TINs compared to samples sequenced on all other dates (Fig. 407 4C-E; ANOVA; $P<0.01)$. TINs quantify the uniformity of coverage across transcripts and are 408 informative as a measure of in vitro RNA degradation, which likely suggests that hybrid 
craniofacial samples experienced more degradation than other samples prior to sequencing.

410 Indeed, lower TIN was significantly correlated with a lower number of normalized counts across

411 samples (Fig. 4F; linear regression; $P=2.0 \times 10^{-5}$ ). Given that hybrid craniofacial samples

412 showed lower TINs and lower normalized counts (Fig. 4C and D), we expected to see a bias

413 toward underexpressed genes in hybrids relative to parental species. Instead, we found

414 approximately the same number of genes overexpressed in hybrids $(25.83 \%)$ as there were genes 415 underexpressed (25.77\%; Fig. 1F and 3B).

416 Overall, we found that our estimate of the proportion of genes misexpressed in 17-20 dpf

417 hybrid craniofacial tissue (51.6\%) may be biased due to differences in the number of duplicate

418 reads produced by two different library preparation methods (Fig. 4E). We quantified this bias by

419 measuring hybrid misexpression between samples collected at an earlier developmental stage

420 and found that $19.1 \%$ of genes were misexpressed in 17-20 dpf hybrid craniofacial tissues after

421 correcting for library preparation biases (Fig. S5). We found that 17-20 dpf hybrid craniofacial

422 tissues likely experienced more in vitro RNA degradation than other samples, but this did not

423 produce a bias toward more genes showing underdominant expression in hybrids (Fig. 3B).

\section{Compensatory variation underlies misexpression in hybrids}

426 If a gene shows similar gene expression levels between parental species but shows biased allelic

427 expression only in hybrids, it may be regulated by compensatory variation, and such genes are

428 likely to be misexpressed in hybrids (Landry et al. 2005; Goncalves et al. 2012). We identified

42915,429 phased heterozygous sites across all $8 \mathrm{dpf}$ and 17-20 dpf individuals with sequencing

430 coverage $\geq 20 \times$ that fell within 2,761 (8 dpf) and 1,911 (17-20 dpf) genes used for differential

431 expression analyses. We estimated allele specific expression (ASE) for these genes and paired

432 these data with patterns of differential expression between parental species to identify genes

433 controlled compensatory variation.

434 We measured ASE across sites within 2,770 genes that showed no difference in 435 expression between generalists and molluscivores at $8 \mathrm{dpf}$. We found 157 genes (5.4\%) that were 436 likely regulated by compensatory mechanisms, which showed ASE only in hybrids and were not 
437 differentially expressed between generalists and molluscivores. Of these, nine genes $(0.33 \%)$

438 also showed misexpression in hybrids (Fig. 5A and C).

We also measured ASE across sites within 2,387 genes that showed no difference in 440 expression between generalists and molluscivores at 17-20 dpf. We found 1080 genes (44.81\%)

441 that were likely regulated by compensatory mechanisms. In support of this wide-spread

442 compensatory regulation, 581 of these 1080 genes $(53.8 \%)$ also showed misexpression in hybrids

443 (Fig. 5B and D).

We found many more genes showing ASE in 17-20 dpf hybrid craniofacial tissue than any other samples (Fig. 6A; ANOVA, $P=2.81 \times 10^{-5}$ ). Since misexpression is expected in

446 hybrids when gene expression is controlled by compensatory variation between parental species

447 (Landry et al. 2005; Bedford and Hartl 2009; Goncalves et al. 2012), the high number of genes

448 showing compensatory regulation and high number of genes showing ASE in hybrids supports

449 the validity of extensive misexpression in 17-20 dpf hybrid craniofacial tissue. We likely

450 overestimated the amount of misexpression in this tissue because hybrids were sequenced using

451 a different library preparation kit than parental species (see above). However, ASE was

452 estimated by examining allelic ratios in individual samples and should not suffer from this bias.

453 17-20 dpf hybrid craniofacial tissue was sequenced at the same facility using the same library

454 preparation kit as all of the $8 \mathrm{dpf}$ samples (Table 1 and S1), yet we only found a high number of 455 genes showing ASE in the 17-20 dpf hybrids (Fig 6A).

456 We tested whether this pattern might be due to higher rates of in vitro degradation in 457 hybrid samples (reflected by low TINs), which could increase variance in the abundance of reads 458 at heterozygous sites and bias ASE estimates. Lower TIN was correlated with higher ASE (Fig.

$4596 \mathrm{D}$; linear regression; $P=9.04 \times 10^{-14}$ ). This correlation persisted when $17-20 \mathrm{dpf}$ hybrid

460 craniofacial samples were excluded from the model (Fig. 6E; linear regression; $P=0.034$ ).

461 However, the proportion of genes showing ASE was much higher in 17-20 dpf hybrid

462 craniofacial samples than predicted by the latter linear model. Even the lowest TIN for a 17-20

463 dpf hybrid sample (32.68) predicted a much lower range of genes showing ASE (8.2\% - $14.1 \%)$

464 compared to the observed range (32.8\% - 51.6\%). We also estimated ASE again with a higher

465 coverage threshold ( $>=100$ counts supporting each heterozygous allele) to reduce the chances of 
466 increased variance affecting binomial tests and still found that hybrid craniofacial samples

467 showed more ASE than other samples (Fig. 6B; ANOVA, $P=3.85 \times 10^{-4}$ ).

469 Discussion

470 Molluscivores show extreme craniofacial divergence relative to their generalist sister species,

471 exhibiting a novel maxillary protrusion and short robust jaws (Fig 1A; Martin and Wainwright

472 2013a; Hernandez et al. 2018). Given the extreme craniofacial divergence observed between

473 molluscivores and their generalist sister-species, we might expect to find genes expressed in

474 hybrids outside the range of either parent species as a result of discordance between alternatively

475 coadapted genes in networks shaping divergent craniofacial morphologies. However, genetic

476 divergence between generalists and molluscivores is low, with only 79 SNPs fixed between

477 species (genome-wide average $\mathrm{F}_{\mathrm{st}}=0.08, \mathrm{D}_{\mathrm{xy}}=0.00166$ (McGirr and Martin 2017; McGirr and

478 Martin 2018)). Despite this low genetic divergence and ongoing gene flow between species, we

479 found misexpression in hybrids at two developmental stages and tissue types. We also measured

480 allele specific expression (ASE) for genes expressed in hybrids and parental species and found

481 evidence for compensatory divergence influencing hybrid misexpression at both developmental 482 stages.

\section{Hybrid misexpression during juvenile development}

485 While many studies on hybrid misexpression search for regulatory divergence in 'speciation 486 genes' associated with sterility and inviability (Malone and Michalak 2008; Renaut et al. 2009;

487 Davidson and Balakrishnan 2016), our results highlight the importance of considering 488 misexpression over multiple early developmental stages and in the context of adaptive 489 morphological traits. We found evidence of misexpression in $8 \mathrm{dpf}$ whole-larvae hybrid tissues 490 (2.1\% of genes) and in 17-20 dpf hybrid craniofacial tissues (19.1\% of genes after correcting for 491 bias due to library preparation method).

492 There are several reasons why we might expect to find a higher proportion of genes 493 misexpressed in 17-20 dpf hybrid craniofacial tissues relative to $8 \mathrm{dpf}$ whole-larvae tissues. The 
494 molluscivore shows exceptional rates of morphological diversification, particularly in

495 craniofacial traits (Martin and Wainwright 2011). Perhaps 17-20 dpf is a crucial developmental

496 window when gene networks shaping these traits become extensively misregulated in hybrids. It

497 is just after this stage that the relative length of the premaxilla, maxilla, palatine, and lower jaw

498 tend to increase more for generalists than molluscivores (Lencer et al. 2016). It is also possible

499 that regulatory changes are compounded throughout development and have cascading effects,

500 resulting in higher rates of misexpression in later stages. Finally, some of the increased

501 misexpression in hybrid craniofacial tissue can likely be attributed to our sampling design. We

502 found that hybrid craniofacial samples showed lower TINs and lower normalized counts (Fig. 4A

503 and D), suggesting that these samples may have experienced more in vitro RNA degradation

504 than other samples (Wang et al. 2016). However, under this scenario, we would expect to see a

505 bias toward lower gene expression in hybrids relative to parental species. Alternatively, we

506 found approximately the same number of genes overexpressed in hybrids (25.83\%) as there were

507 genes underexpressed (25.77\%), suggesting that many genes were overexpressed in hybrids

508 despite potential RNA degradation.

509 We found roughly twice the amount of misexpression in hybrid craniofacial tissues

510 compared to a study of misexpression in whole-body tissue that measured gene expression in $\mathrm{F}_{1}$

511 hybrids generated between benthic and limnetic lake whitefish (Renaut et al. 2009). These

512 populations also diverged within the past 10 kya and occupy different habitats within lakes

513 (Renaut et al. 2009; Bernatchez 2004). We also found that genes showing underdominance in

514 hybrids showed a higher magnitude of differential expression compared to those showing

515 overdominance in $8 \mathrm{dpf}$ and 17-20 dpf tissues (Fig. S4), a pattern that has also been observed in

516 whitefish (Renaut and Bernatchez 2011) and a generalist/specialist Drosophila species pair

517 (McManus et al. 2010).

\section{The consequences of hybrid misexpression}

520 It is unclear whether such extensive gene misexpression in hybrid craniofacial tissues might

521 contribute to intrinsic post-zygotic isolation between generalists and molluscivores. $\mathrm{F}_{2}$ hybrids

522 exhibiting intermediate and transgressive craniofacial phenotypes showed reduced survival and

523 growth rates in the wild relative to $\mathrm{F}_{2}$ hybrids resembling parental species (Martin and 
524 Wainwright 2013b; Martin 2016), but short-term experiments measuring $\mathrm{F}_{2}$ hybrid survival in

525 the lab did not find any evidence of reduced survival for hybrids with intermediate phenotypes

526 (Martin and Wainwright 2013b). This was interpreted as evidence that complex fitness

527 landscapes measured in field enclosures on San Salvador with multiple peaks corresponding to

528 the generalist and molluscivore phenotypes were due to competition and foraging ability in the

529 wild (i.e. extrinsic reproductive isolation). However, additional analyses of these data suggest

530 that absolute performance of hybrids may also play a role in their survival. The most

531 transgressive hybrid phenotypes exhibited the lowest fitness, contrary to expectations from

532 negative frequency-dependent disruptive selection (Martin 2016). It is still possible that intrinsic

533 and extrinsic incompatibilities interact such that gene misexpression weakens performance more

534 in the wild than in the lab. However, note that $F_{1}$ hybrids used in this study would fall within an

535 intermediate phenotypic position relative to parental trophic morphology whereas field

536 experiments used $\mathrm{F}_{2}$ and later generation hybrid intercrosses and backcrosses.

\section{Hybrid misexpression is controlled by compensatory divergence}

539 When an optimal level of gene expression is favored by stabilizing selection, compensatory

540 variation can accumulate between species and cause misexpression in hybrids (Landry et al.

541 2005; Bedford and Hartl 2009). We combined results from differential expression analyses with

542 allele specific expression (ASE) results to identify genes controlled by compensatory regulatory

543 divergence between generalists and molluscivores. In $8 \mathrm{dpf}$ whole-larvae tissue, we found 5.4\%

544 of genes controlled by compensatory regulation (Fig. 5B). The low amount of genes controlled

545 by compensatory regulation was reflected by the low amount of genes misexpressed in $8 \mathrm{dpf}$

546 hybrids (2.1\%). In 17-20 dpf hybrid craniofacial tissues, we found $44.81 \%$ of genes controlled by

547 compensatory regulation (Fig. 5B). The large number of genes controlled by compensatory

548 regulation is consistent with the extensive misexpression observed in hybrid craniofacial tissue,

549 and the majority of genes showing signs of compensatory regulation were also misexpressed in

550 hybrids (53.8\%). This independent line of evidence supporting misexpression in 17-20 dpf

551 hybrid craniofacial tissue was not biased by differences in library preparation methods because

552 allele specific expression was estimated by measuring allelic ratios in individual samples. 17-20

553 dpf hybrid craniofacial tissue was sequenced at the same facility using the same library 
554 preparation kit as the $8 \mathrm{dpf}$ samples, yet we only found a high number of genes showing ASE in

555 the 17-20 dpf hybrids (Fig. 6). These results are also in line with studies finding widespread

556 compensatory evolution in other systems with greater divergence times between species (Landry

557 et al. 2005, 2007; Takahasi et al. 2011; Goncalves et al. 2012; Bell et al. 2013; Mack and

558 Nachman 2017; but also see Fraser 2018).

Conclusion

561 We found hybrid misexpression in both whole-larvae tissues cranial tissues sampled at early

562 developmental stages. This points to divergent evolution of developmental networks shaping

563 novel traits in the molluscivore. It is unclear whether such misexpression causes intrinsic

564 incompatibilities in hybrids within this recent adaptive radiation. Investigating mechanisms

565 regulating gene expression between generalists and molluscivores that result in hybrid

566 misexpression will shed light on whether the variants shaping novel traits may also contribute to

567 reproductive isolation. Examining misexpression across multiple early developmental stages in

568 the context of developing tissues that give rise to adaptive traits can paint a more complete

569 picture of genetic incompatibilities that distinguish species.

\section{Data Availability}

572 All transcriptomic raw sequence reads are available as zipped fastq files on the NCBI BioProject 573 database. Accession: PRJNA391309. Title: Craniofacial divergence in Caribbean Pupfishes.

\section{Acknowledgements}

576 This study was funded by the University of North Carolina at Chapel Hill and the Miller Institute

577 for Basic Research in the Sciences to CHM. We thank Daniel Matute, Emilie Richards, Michelle

578 St. John, and Sara Suzuki for valuable discussions and computational assistance; Emilie Richards

579 for photographs of specimens in Figure 1; The Vincent J. Coates Genomics Sequencing

580 Laboratory at the University of California, Berkeley and the High-Throughout Sequencing

581 Facility at the University of North Carolina, Chapel Hill for performing RNA library prep and 
582 Illumina sequencing; the Gerace Research Centre for accommodation; and the Bahamian

583 government BEST Commission for permission to conduct this research.

Author Contributions

586 JAM wrote the manuscript, extracted the RNA samples, and conducted all bioinformatic

587 analyses. Both authors contributed to the conception and development of the ideas and revision 588 of the manuscript.

\section{Competing interests}

591 We declare we have no competing interests.

\section{References}

595 Ashburner, M., C. A. Ball, J. A. Blake, D. Bolstein, H. Butler, J. M. Cherry, A. P. Davis, K. 596 Dolinski, S. S. Dwight, J. T. Eppig, et al. 2000. Gene ontology: tool for the unification of biology. Nat. Genet. 25:25-29.

Alberti, A., C. Belser, S. Engelen, L. Bertrand, C. Orvain, L. Brinas, C. Cruaud, L. Giraut, C. Da Silva, C. Firmo, J. M. Aury, and P. Wincker. 2014. Comparison of library preparation methods reveals their impact on interpretation of metatranscriptomic data. BMC Genomics 15:1-13.

Bedford, T., and D. L. Hartl. 2009. Optimization of gene expression by natural selection. Proc. allele-specific expression, hybrid effects, and regulatory divergence in hybrids compared with their parents from natural populations. Genome Biol. Evol. 5:1309-1323.

607 Biosystems, K. 2014. Technical Data Sheet KAPA Stranded mRNA-Seq Kit. 1-16. 
Carroll, S. B. 2008. Perspective Evo-Devo and an Expanding Evolutionary Synthesis $\square$ : A Genetic Theory of Morphological Evolution. 25-36.

Coolon, J. D., C. J. Mcmanus, K. R. Stevenson, J. D. Coolon, C. J. Mcmanus, K. R. Stevenson, B. R. Graveley, and P. J. Wittkopp. 2014. Tempo and mode of regulatory evolution in variation in mouse genes. Nat. Genet. 32:432-437.

Coyne, J. A. \& O. 2004. Speciation. Sunderland, MA Sinauer Assoc.

Davidson, J. H., and C. N. Balakrishnan. 2016. Gene Regulatory Evolution During Speciation in a Songbird. 6:1357-1364.

Denver, D. R., K. Morris, J. T. Streelman, S. K. Kim, M. Lynch, and W. K. Thomas. 2005. The transcriptional consequences of mutation and natural selection in Caenorhabditis elegans. Nat. Genet., doi: 10.1038/ng1554.

DePristo, M. A., E. Banks, R. Poplin, K. V Garimella, J. R. Maguire, C. Hartl, A. A. Philippakis, G. del Angel, M. A. Rivas, M. Hanna, A. McKenna, T. J. Fennell, A. M. Kernytsky, A. Y.

Dobin, A., C. A. Davis, F. Schlesinger, J. Drenkow, C. Zaleski, S. Jha, P. Batut, M. Chaisson,

Ewels, P., S. Lundin, and K. Max. 2016. Data and text mining MultiQC $\square$ : summarize analysis results for multiple tools and samples in a single report. 32:3047-3048.

630 Fang, S., R. Yukilevich, Y. Chen, D. A. Turissini, K. Zeng, I. A. Boussy, and C. I. Wu. 2012.

633 Fraser, H. B. 2018. Improving Estimates of Compensatory cis-trans Regulatory Divergence.

634 Goncalves, A., S. Leigh-Brown, D. Thybert, K. Stefflova, E. Turro, P. Flicek, A. Brazma, D. T. 
Odom, and J. C. Marioni. 2012. Extensive compensatory cis-trans regulation in the evolution of mouse gene expression. Genome Res. 22:2376-2384.

637 Haerty, W., and R. S. Singh. 2006. Gene regulation divergence is a major contributor to the evolution of Dobzhansky-Muller incompatibilities between species of Drosophila. Mol. Biol. Evol. 23:1707-1714.

Hernandez, L. P., D. Adriaens, C. H. Martin, P. C. Wainwright, B. Masschaele, and M. Dierick. 2018. Building trophic specializations that result in substantial niche partitioning within a young adaptive radiation. J. Anat. 232:173-185.

Hodgins-Davis, A., D. P. Rice, J. P. Townsend, and J. Novembre. 2015. Gene expression evolves under a house-of-cards model of stabilizing selection. Mol. Biol. Evol. 32:2130-2140.

Holtmeier, C. L. 2001. Heterochrony, maternal effects, and phenotypic variation among sympatric pupfishes. Evolution (N. Y). 55:330-338.

Hughes, K. A., J. F. Ayroles, M. M. Reedy, J. M. Drnevich, K. C. Rowe, E. A. Ruedi, C. E. Cáceres, and K. N. Paige. 2006. Segregating variation in the transcriptome: Cis regulation and additivity of effects. Genetics 173:1347-1355.

Indjeian, V. B., G. A. Kingman, F. C. Jones, C. A. Guenther, J. Grimwood, J. Schmutz, R. M. Compensatory cis-trans evolution and the dysregulation of gene expression in interspecific hybrids of drosophila. Genetics 171:1813-1822.

Lencer, E. S., M. L. Riccio, and A. R. McCune. 2016. Changes in growth rates of oral jaw elements produce evolutionary novelty in bahamian pupfish. J. Morphol. 277:935-947. 
closely related species. 1-33. BMC Genomics.

663 Liao, Y., G. K. Smyth, and W. Shi. 2014. Sequence analysis featureCounts $\square$ : an efficient

664 general purpose program for assigning sequence reads to genomic features. 30:923-930.

665 Mack, K. L., and M. W. Nachman. 2017. Gene Regulation and Speciation. Trends Genet. 33:68-

666 80.

Malone, J. H., and P. Michalak. 2008. Gene expression analysis of the ovary of hybrid females of Xenopus laevis and X. muelleri. BMC Evol. Biol. 8.

669 Marsden, C. D., Y. Lee, K. Kreppel, A. Weakley, A. Cornel, H. M. Ferguson, E. Eskin, and G.

Martin, C. H. 2016. Context-dependence in complex adaptive landscapes $\square$ : frequency and traitdependent selection surfaces within an adaptive radiation of Caribbean pupfishes. Evolution

Martin, C. H., and L. C. Feinstein. 2014. Novel trophic niches drive variable progress towards ecological speciation within an adaptive radiation of pupfishes. Mol. Ecol. 23:1846-1862.

677 Martin, C. H., and P. C. Wainwright. 2013a. A remarkable species flock of Cyprinodon pupfishes endemic to San Salvador Island, Bahamas. Bull. Peabody Museum Nat. Hist. $54: 231-241$.

Martin, C. H., and P. C. Wainwright. 2013b. Multiple fitness peaks on the adaptive landscape

Martin, C. H., and P. C. Wainwright. 2013c. On the measurement of ecological novelty: scalemorphological diversification in two adaptive radiations of Cyprinodon pupfish. Evolution

688 Mcgirr, J. A., and C. H. Martin. 2016. Novel Candidate Genes Underlying Extreme Trophic 
Specialization in Caribbean Pupfishes. 34:873-888.

McGirr, J. A., and C. H. Martin. 2018. Parallel evolution of gene expression between trophic specialists despite divergent genotypes and morphologies. Evol. Lett. 62-75.

McGirr, J. A., C. H. Martin, and D. Agashe. 2017. Novel candidate genes underlying extreme trophic specialization in caribbean pupfishes. Mol. Biol. Evol. 34.

McManus, C. J., J. D. Coolon, M. O. Duff, J. Eipper-Mains, B. R. Graveley, and P. J. Wittkopp. 2010. Regulatory divergence in Drosophila revealed by mRNA-seq. Genome Res. 20:816825.

Michalak, P., and M. A. F. Noor. 2004. Association of misexpression with sterility in hybrids of

Mylroie, J.E, Hagey, F. M. 1995. Terrestrial and Shallow Marine Geology of the Bahamas and Bermuda. Geological Society of America, Boulder, CO.

Ortíz-Barrientos, D., B. A. Counterman, and M. A. F. Noor. 2007. Gene expression divergence

Presgraves, D. C. 2003. A fine-scale genetic analysis of hybrid incompatibilities in lemph\{Drosophila\}. Genetics 163:955-972. Hartl. 2004. Anomalies in the Expression Profile of Interspecific Hybrids of Drosophila and the origin of hybrid dysfunctions. Genetica 129:71-81.

melanogaster and Drosophila simulans Anomalies in the Expression Profile of Interspecific Hybrids of Drosophila melanogaster and Drosophila simulans. 373-379.

Renaut, S., and L. Bernatchez. 2011. Transcriptome-wide signature of hybrid breakdown associated with intrinsic reproductive isolation in lake whitefish species pairs (Coregonus spp. Salmonidae). Heredity (Edinb). 106:1003-1011. Nature Publishing Group.

715 Roberge, C., É. Normandeau, S. Einum, H. Guderley, and L. Bernatchez. 2008. Genetic 
consequences of interbreeding between farmed and wild Atlantic salmon: Insights from the transcriptome. Mol. Ecol. 17:314-324.

Rockman, M. V., and L. Kruglyak. 2006. Genetics of global gene expression. Nat. Rev. Genet. $7: 862-872$.

Rottscheidt, R., and B. Harr. 2007. Extensive additivity of gene expression differentiates subspecies of the house mouse. Genetics 177:1553-1567.

722 Schumer, M., R. Cui, D. L. Powell, R. Dresner, G. G. Rosenthal, and P. Andolfatto. 2014. Highresolution mapping reveals hundreds of genetic incompatibilities in hybridizing fish species. Elife 2014:1-21.

Signor, S. A., and S. V. Nuzhdin. 2018. The Evolution of Gene Expression in cis and trans. Trends Genet. 1-13. Elsevier Ltd.

Society, R. S. 2017. Controlling the False Discovery Rate $\square$ : A Practical and Powerful Approach to Multiple Testing Author ( s ): Yoav Benjamini and Yosef Hochberg Source $\square$ : Journal of

Sweigart, A. L., L. Fishman, and J. H. Willis. 2006. A simple genetic incompatibility causes

Takahasi, K. R., T. Matsuo, and T. Takano-Shimizu-Kouno. 2011. Two types of cis-trans the Royal Statistical Society . Series B ( Methodological ), Vol . 57 , No . 1 Published by $\square$ :

True, J. R., and E. S. Haag. 2001. Developmental system drift and flexibility in evolutionary trajectories. Evol. Dev. 3:109-119.

738 Turner, B. J., D. D. Duvernell, T. M. Bunt, and M. G. Barton. 2008. Reproductive isolation 739 among endemic pupfishes (Cyprinodon) on San Salvador Island, Bahamas: Microsatellite $740 \quad$ evidence. Biol. J. Linn. Soc. 95:566-582.

741 Van Dijk, E. L., Y. Jaszczyszyn, and C. Thermes. 2014. Library preparation methods for next742 generation sequencing: Tone down the bias. Exp. Cell Res. 322:12-20. Elsevier. 
743 Wainwright, P. C., and B. A. Richard. 1995. Predicting patterns of prey use from morphology of $744 \quad$ fishes. Environ. Biol. Fishes 44:97-113.

745 Wang, L., J. Nie, H. Sicotte, Y. Li, J. E. Eckel-Passow, S. Dasari, P. T. Vedell, P. Barman, L.

746 Wang, R. Weinshiboum, J. Jen, H. Huang, M. Kohli, and J. P. A. Kocher. 2016. Measure transcript integrity using RNA-seq data. BMC Bioinformatics 17:1-16. BMC

$748 \quad$ Bioinformatics.

749 Wang, L., S. Wang, and W. Li. 2012. RSeQC: quality control of RNA-seq experiments.

750 Bioinforma. Oxford Engl., doi: 10.1093/bioinformatics/bts356.

751 West, R. J. D., and A. Kodric-Brown. 2015. Mate Choice by Both Sexes Maintains Reproductive 752 Isolation in a Species Flock of Pupfish (Cyprinodon spp) in the Bahamas. Ethology $753 \quad 121: 793-800$.

754 Wittkopp, P. J., B. K. Haerum, and A. G. Clark. 2012. Evolutionary changes in cis and trans 755 gene regulation. 1678:2010-2012.

756 Wittkopp, P. J., B. K. Haerum, and A. G. Clark. 2004. Evolutionary changes in cis and trans 757 gene regulation. Nature, doi: 10.1038/nature02698.

758 Wolf, J. B. W., J. Lindell, and N. Backstrom. 2010. Speciation genetics: current status and 759 evolving approaches. Philos. Trans. R. Soc. B Biol. Sci. 365:1717-1733. 
776 Table 1. Sampling design for mRNA sequencing. Parental fishes crossed to produce larvae for 777 sequencing were either wild-caught $\left(\mathrm{F}_{0}\right)$ or lab-raised over $n$ generations (indicated by $\mathrm{F}_{n}$ ).

\begin{tabular}{|c|c|c|c|c|c|}
\hline mothers & fathers & $\begin{array}{l}\text { offspring } \\
\text { sampled }\end{array}$ & $\begin{array}{c}\text { lake } \\
\text { population }\end{array}$ & $\begin{array}{l}\text { sequencing } \\
\text { round }\end{array}$ & $\begin{array}{l}\text { stage } \\
\text { (dpf) }\end{array}$ \\
\hline $\mathrm{F}_{0}$ generalist & $\mathrm{F}_{0}$ molluscivore & 3 hybrids & osprey lake & 4 & 8 \\
\hline $\mathrm{F}_{0}$ generalists & $\mathrm{F}_{0}$ generalists & 3 generalists & osprey lake & 3 & 8 \\
\hline $\mathrm{F}_{0}$ molluscivores & $\mathrm{F}_{0}$ molluscivores & 3 molluscivores & osprey lake & 3 & 8 \\
\hline $\mathrm{F}_{0}$ generalists & $\mathrm{F}_{0}$ generalists & 3 generalists & crescent pond & 3 & 8 \\
\hline $\mathrm{F}_{0}$ molluscivores & $\mathrm{F}_{0}$ molluscivores & 3 molluscivore & crescent pond & 4 & 8 \\
\hline $\mathrm{F}_{1}$ generalists & $\mathrm{F}_{1}$ generalists & 3 generalists & little lake & 1 & $8-10$ \\
\hline $\mathrm{F}_{2}$ molluscivores & $\mathrm{F}_{2}$ molluscivores & 3 molluscivores & little lake & 1 & $8-10$ \\
\hline $\mathrm{F}_{2}$ generalists & $\mathrm{F}_{2}$ generalists & 3 generalists & crescent pond & 1 & $8-10$ \\
\hline $\mathrm{F}_{2}$ molluscivores & $\mathrm{F}_{2}$ molluscivores & 3 molluscivores & crescent pond & 1 & $8-10$ \\
\hline $\mathrm{F}_{2}$ generalist & $\mathrm{F}_{3}$ molluscivore & 4 hybrids & little lake & 2 & $17-20$ \\
\hline $\mathrm{F}_{1}$ generalists & $\mathrm{F}_{1}$ generalists & 3 generalists & little lake & 1 & $17-20$ \\
\hline $\mathrm{F}_{2}$ molluscivores & $\mathrm{F}_{2}$ molluscivores & 3 molluscivores & little lake & 1 & $17-20$ \\
\hline $\mathrm{F}_{2}$ generalists & $\mathrm{F}_{2}$ generalists & 3 generalists & crescent pond & 1 & $17-20$ \\
\hline $\mathrm{F}_{2}$ molluscivores & $\mathrm{F}_{2}$ molluscivores & 3 molluscivores & crescent pond & 1 & $17-20$ \\
\hline
\end{tabular}


bioRxiv preprint doi: https://doi.org/10.1101/372912; this version posted February 5, 2019. The copyright holder for this preprint (which was not certified by peer review) is the author/funder, who has granted bioRxiv a license to display the preprint in perpetuity. It is made available under aCC-BY-NC-ND 4.0 International license. 

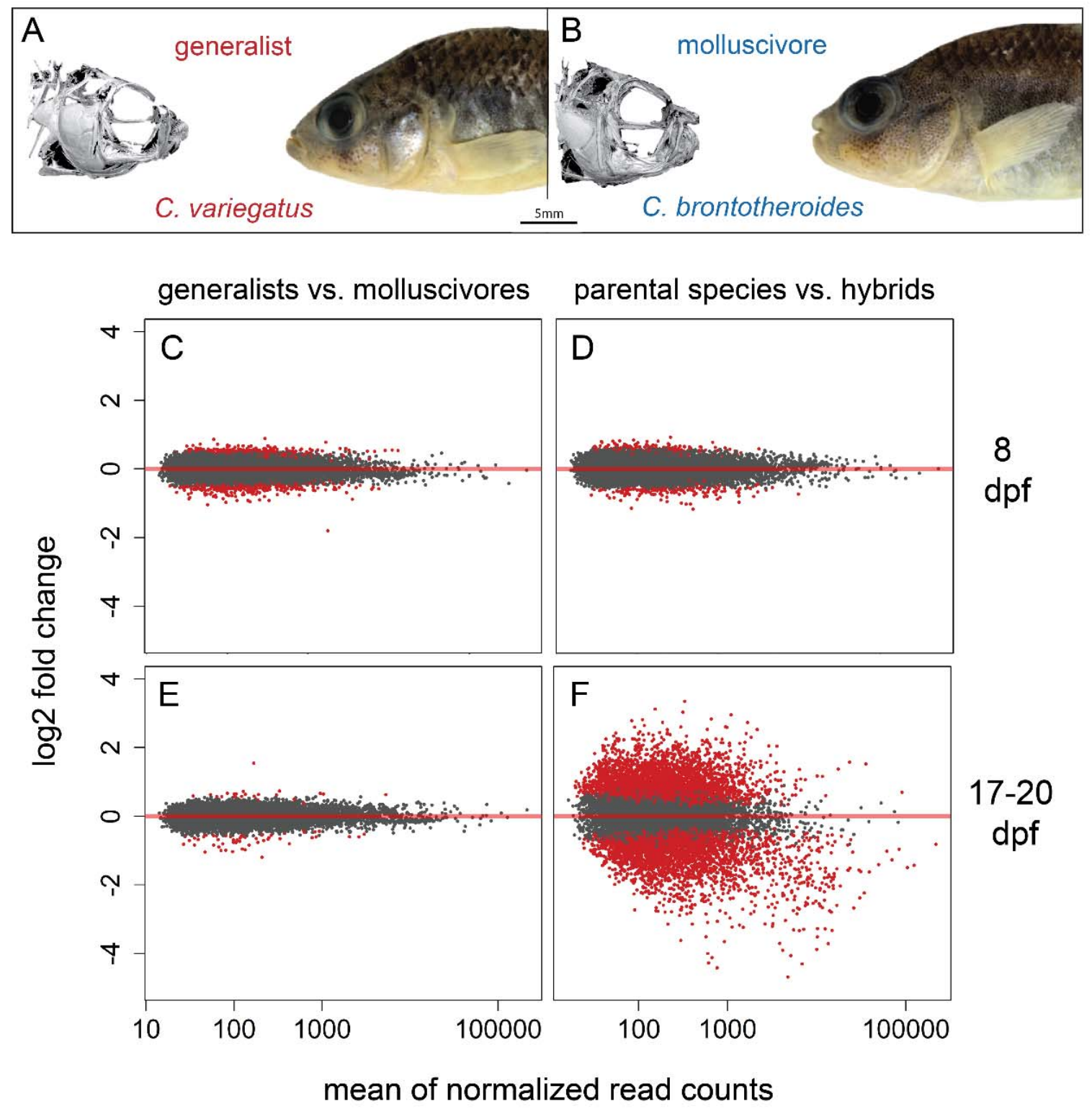

Fig 1. Extensive misexpression in $\mathbf{F}_{\mathbf{1}}$ hybrid craniofacial tissues. A) Cyprinodon variegatus the generalist. B) C. brontotheroides - the molluscivore ( $\mu \mathrm{CT}$ scans of the cranial skeleton of each species modified from Hernandez et al. 2018). Variation in gene expression between generalists vs. molluscivores at $8 \mathrm{dpf}$, D) parental species vs. hybrids at $8 \mathrm{dpf}$, E) generalists vs. molluscivores at 17-20 dpf, and F) parental species vs. hybrids at 17-20 dpf. Red points indicate genes detected as differentially expressed at $5 \%$ false discovery rate with Benjamini-Hochberg multiple testing adjustment. Grey points indicate genes showing no significant difference in expression between groups. Red line indicates a $\log _{2}$ fold change of zero between groups. Points above/below the line are upregulated/downregulated in molluscivores relative to generalists (C and $\mathrm{E}$ ) or hybrids relative to parental species (D and F). 

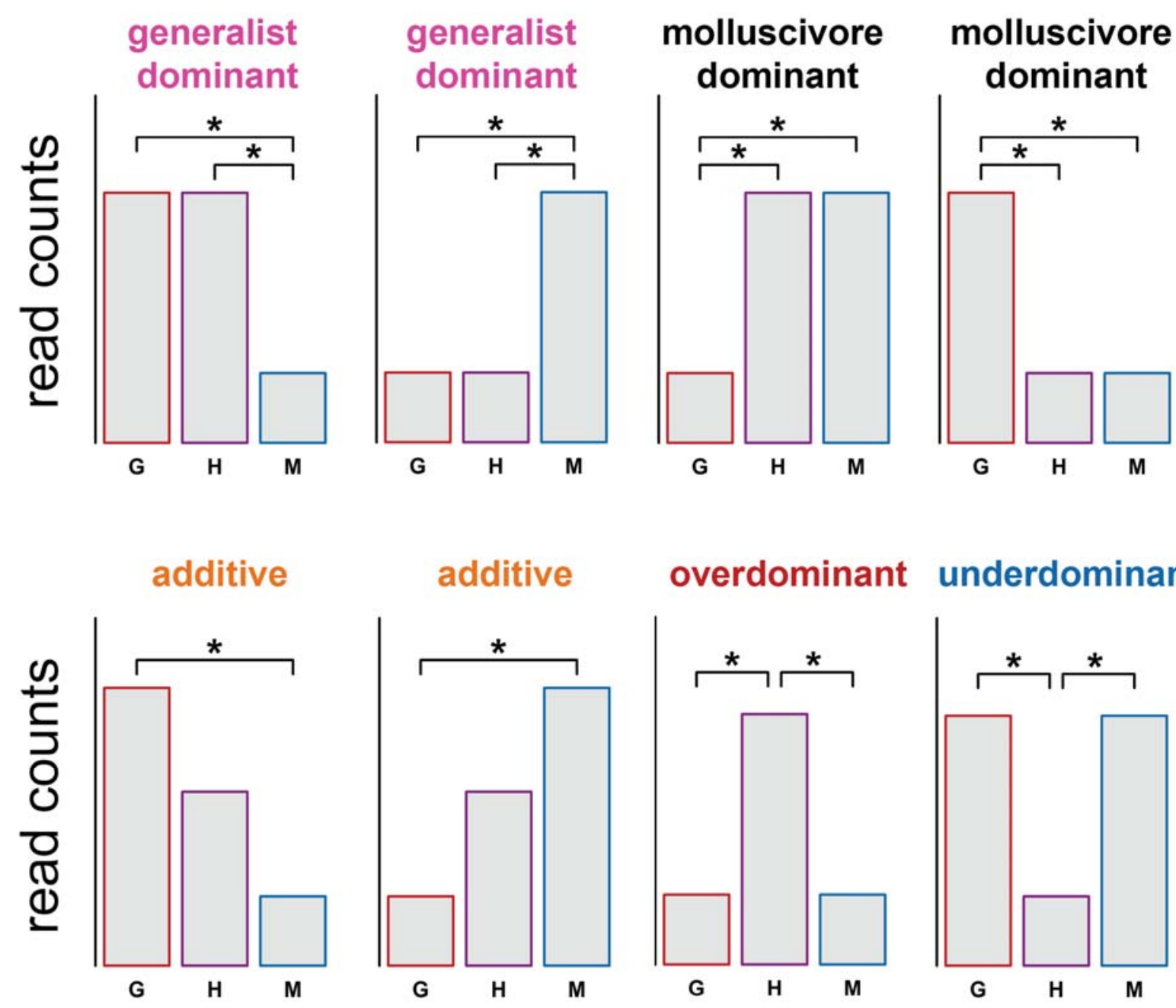

Fig 2. Classifying gene expression inheritance in hybrids. Schematic showing how gene expression inheritance in hybrids was classified. Asterisks indicate significant differential expression between groups. $\mathrm{G}=$ generalists, $\mathrm{H}=$ hybrids, $\mathrm{M}=$ molluscivores. 

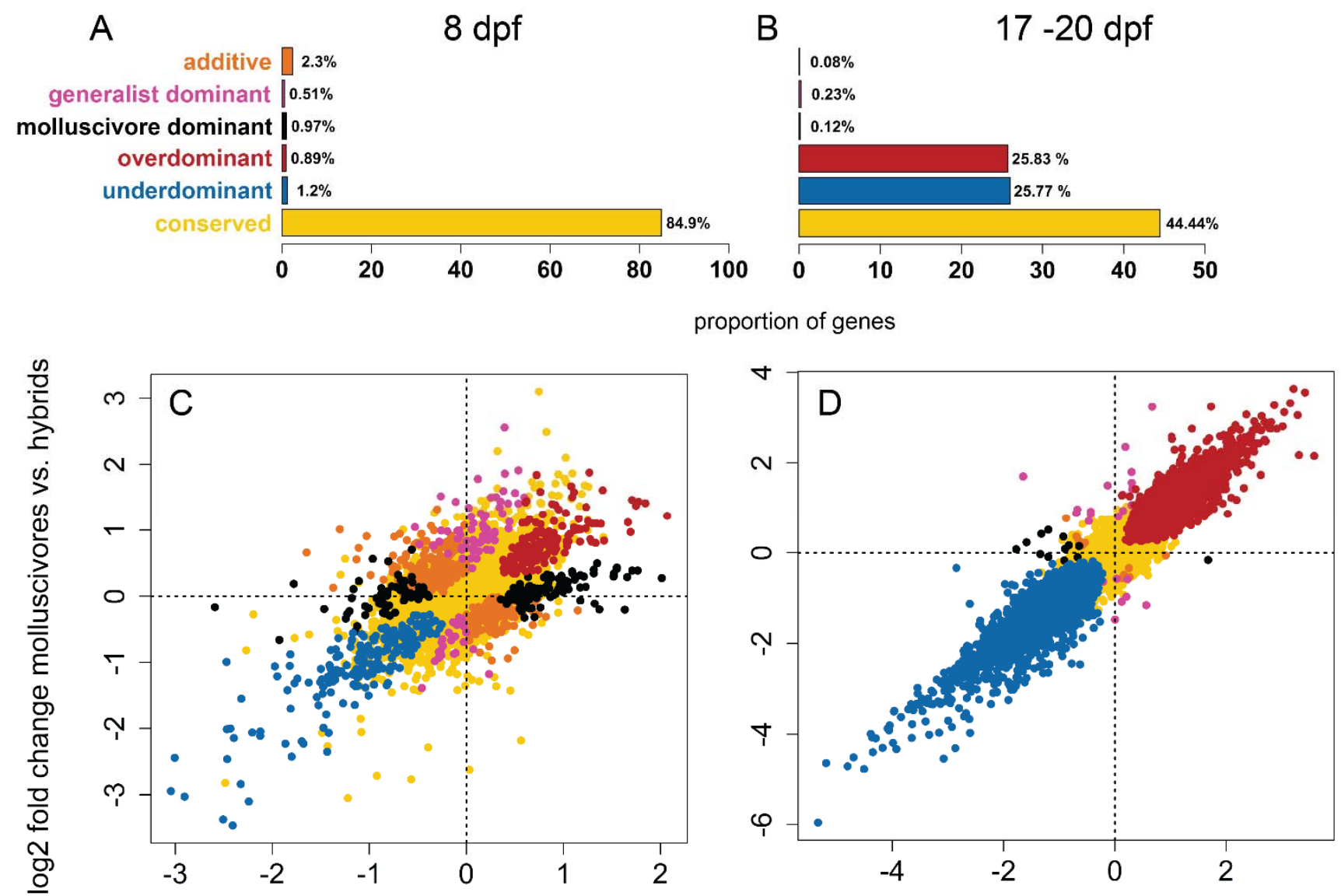

$\log 2$ fold change generalists vs. hybrids

791 Fig 3. Gene expression inheritance in hybrids. The proportion of A) 17,705 and B) 12,769

792 genes showing each class of hybrid gene expression in heritance. $\log _{2}$ fold changes in gene

793 expression between molluscivores vs. hybrids on the y-axis and between generalists vs. hybrids

794 on the $\mathrm{x}$-axis for C) $8 \mathrm{dpf}$ whole-larvae samples and D) 17-20 dpf craniofacial samples. 
A

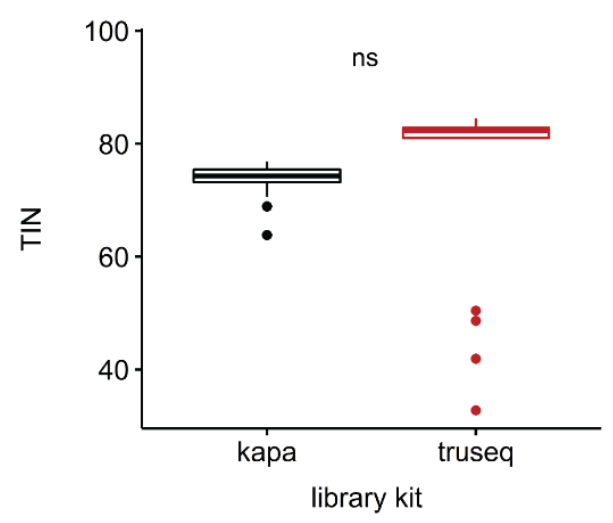

$\mathrm{D}$

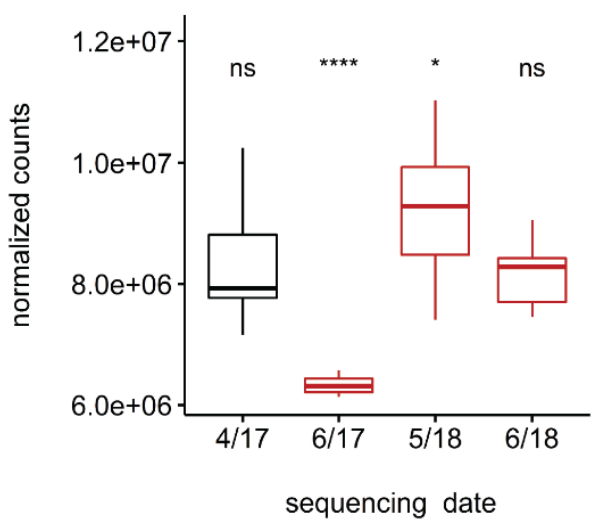

B

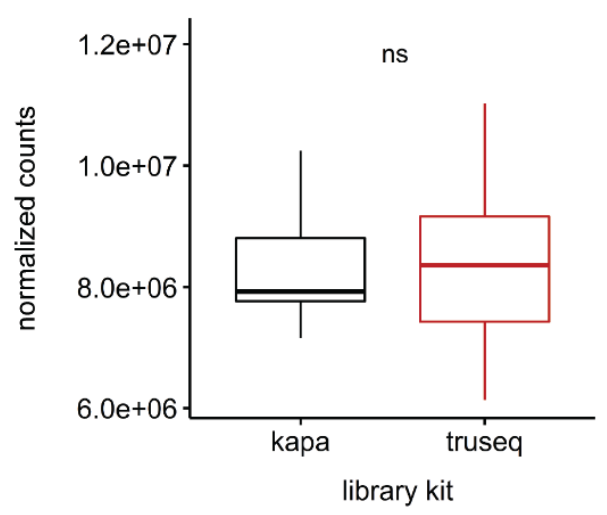

$E$

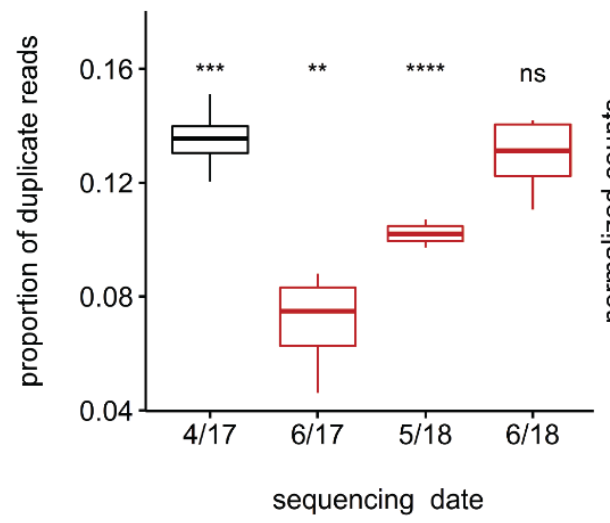

C

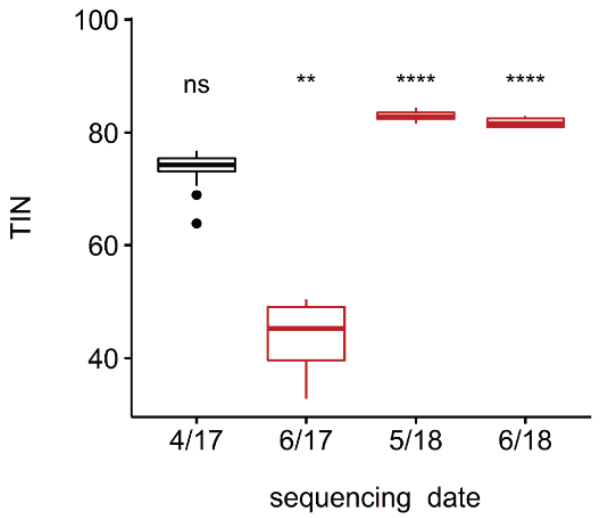

F L Library Prep Kit $\quad \Delta$ hybrid
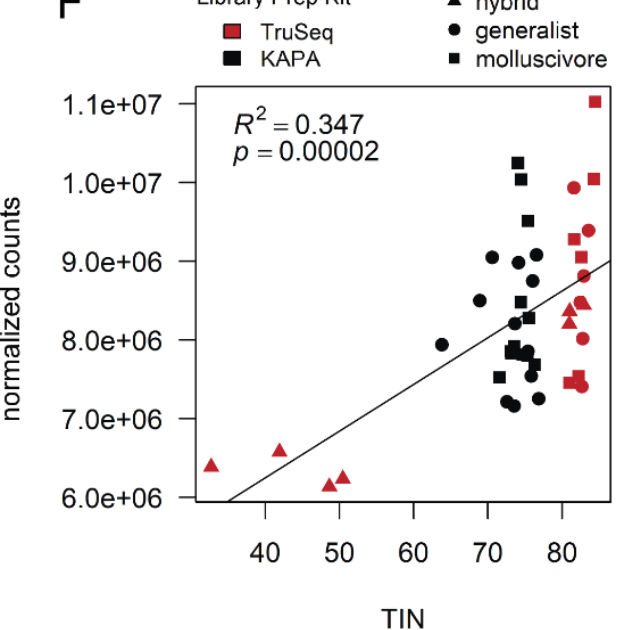

795 Fig 4. Effects of sequencing facility and library preparation kit. Boxplots show samples

796 grouped by library preparation method (A and B) or by the date they were sequenced (C-E) and

797 whether samples were prepared using Truseq stranded mRNA library kits (red) or KAPA

798 stranded mRNA library kits (black). There was no difference in A) median transcript integrity

799 numbers (TIN) or B) number of normalized counts between groups prepared with different

800 library kits (Welch two sample t-test, $P>0.05$ ). 17-20 dpf hybrid craniofacial samples

801 (sequenced 6/17) showed significantly lower C) TIN, D) normalized read counts, and D)

802 proportion of duplicate reads compared to samples sequenced on other dates (ANOVA; $P<$

$8030.0001=* * * *, * * *=0.001, * *=0.01, *=0.05)$. F) Lower TIN was correlated with lower

804 normalized read count. 


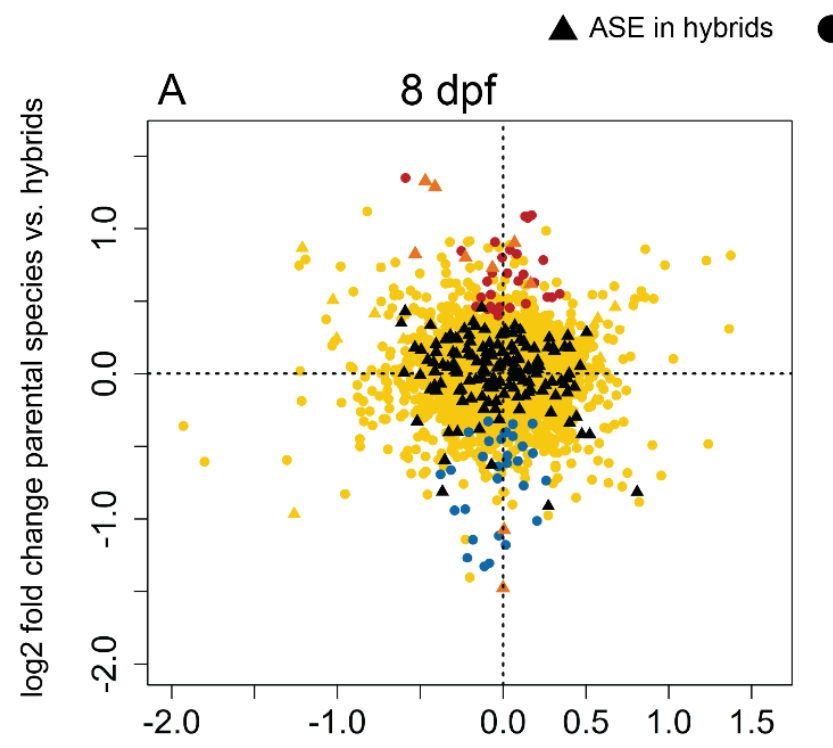

No ASE in hybrids

805

806

807

808

809

810

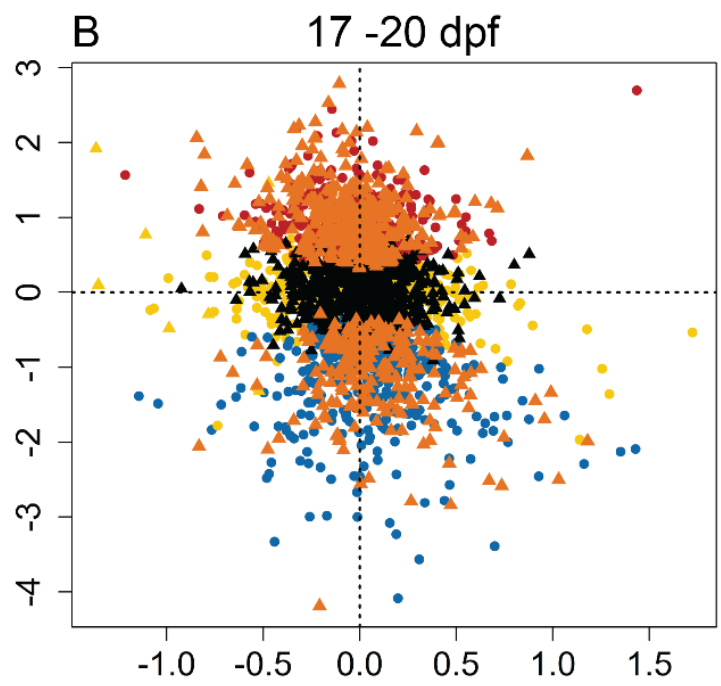

$\log 2$ fold change generalists vs. molluscivores

Fig 5. Compensatory regulation underlying expression divergence between generalists and molluscivores. $\log _{2}$ fold changes in gene expression between parental species vs. hybrids on the $y$-axis and between generalists vs. molluscivores on the x-axis for A) 2,909 genes containing phased heterozygous sites used for allele specific expression (ASE) analyses in $8 \mathrm{dpf}$ wholelarvae samples and B) 2,403 genes containing heterozygous sites in 17-20 dpf craniofacial samples. Triangle points indicate genes showing significant ASE in all hybrids that did not show ASE in generalists or molluscivores. Circle points indicate genes that did not show significant ASE in hybrids or did not show ASE unique to hybrids. Orange = compensatory regulation and hybrid misexpression (genes showing ASE in hybrids, no difference in expression between generalists and molluscivores, and misexpression in hybrids). Black = compensatory regulation (genes showing ASE in hybrids, no difference in expression between generalists and molluscivores). Blue $=$ overdominant (upregulated in hybrids). Red $=$ underdominant (downregulated in hybrids). Yellow = conserved/ambiguous (No difference in expression between parental populations and hybrids). 


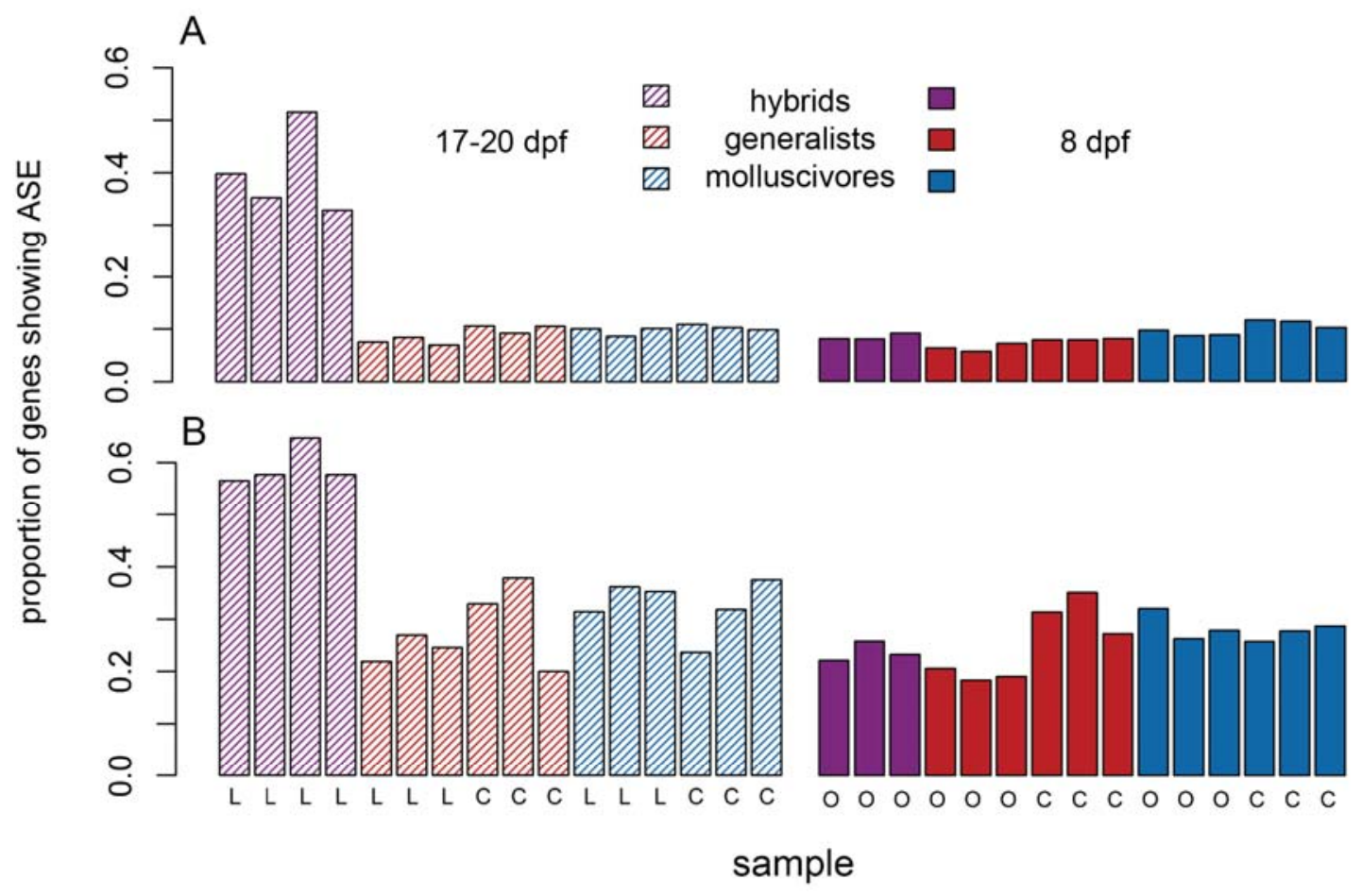

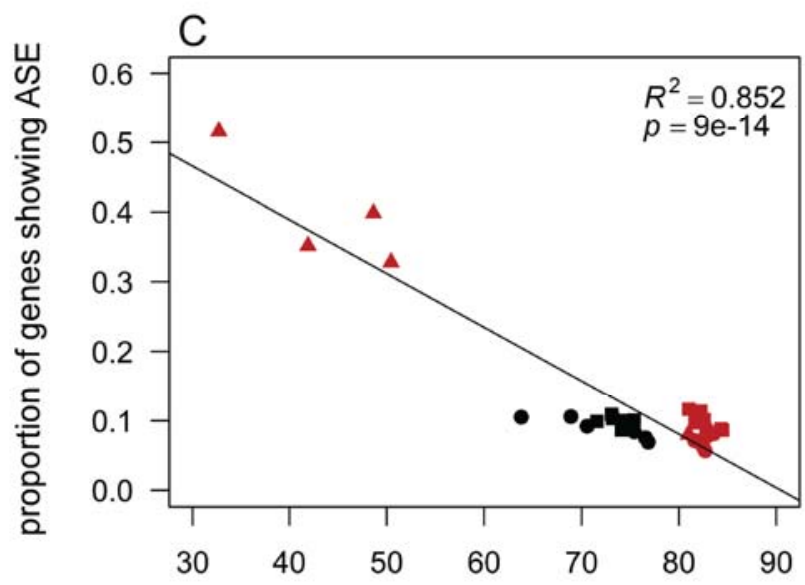

TIN

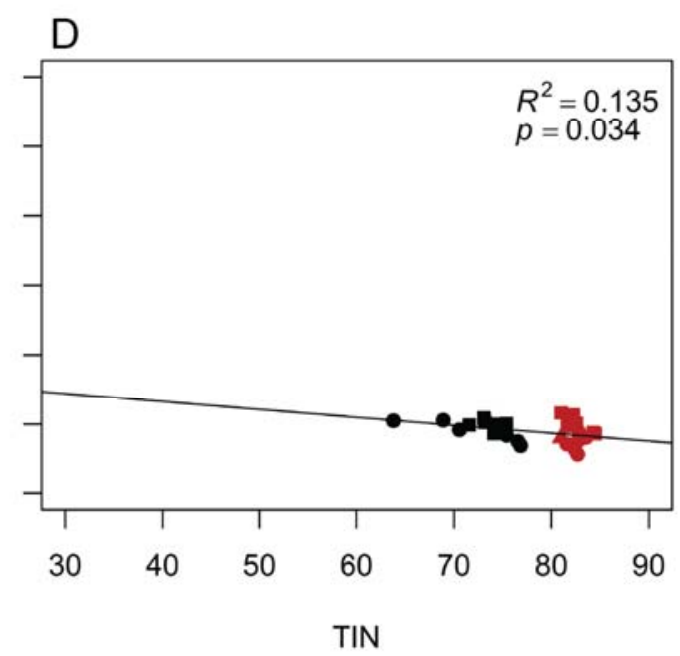

Fig 6. Hybrid craniofacial tissues show high levels of allele specific expression. 17-20 dpf hybrid craniofacial samples (striped purple bars) showed a higher proportion of genes showing significant allele specific expression compared to all other samples using a coverage threshold of A) $\geq 10 \times$ reads supporting each heterozygous allele (ANOVA, $P=2.81 \times 10^{-5}$ ) and $\left.\mathrm{B}\right) \geq 100 \times$ reads supporting each allele (ANOVA, $P=3.85 \times 10^{-4}$ ). $8 \mathrm{dpf}=$ solid, $17-20 \mathrm{dpf}=$ striped; hybrids $=$ purple, generalists $=$ red, molluscivores $=$ blue; $\mathrm{L}=$ Little Lake, $\mathrm{C}=$ Crescent Pond, $\mathrm{O}$ $=$ Osprey Lake. C) TIN was significantly negatively correlated with ASE (linear regression; $P=$ $9.04 \times 10^{-14}$ ). D) This correlation persisted when 17-20 dpf hybrid craniofacial samples were excluded from the linear model (linear regression; $P=0.034$ ). However, the observed proportion of genes showing ASE was much higher in 17-20 dpf hybrid craniofacial samples than predicted by the linear model in D. 